\title{
Differences in Imaging Characteristics of HPV-Positive and HPV-Negative Oropharyngeal Cancers: A Blinded Matched-Pair Analysis
}

\author{
S.C. Cantrell, B.W. Peck, G. Li, Q. Wei, E.M. Sturgis, and L.E. Ginsberg
}

O- $=$

\begin{abstract}
BACKGROUND AND PURPOSE: Human papillomavirus-positive oropharyngeal cancers typically have younger age of onset, limited tobacco exposure, and more favorable prognosis than HPV-negative oropharyngeal cancers. We assessed whether HPV-positive and HPV-negative oropharyngeal cancers have consistent differences in pretreatment imaging characteristics.
\end{abstract}

MATERIALS AND METHODS: A retrospective review of 136 pretreatment CT examinations of paired HPV-positive and HPV-negative oropharyngeal cancers matched for T stage, tumor subsite, and smoking status was performed with the reviewing radiologist blinded to HPV status and clinical stage. Demographic/clinical characteristics and imaging characteristics of primary lesions and metastatic nodal disease were compared by use of Fisher exact testing. The McNemar $\chi^{2}$ test was used for the matched-pair analysis.

RESULTS: By imaging, HPV-negative tumors were more likely to demonstrate invasion of adjacent muscle $(26 \%$ versus $6 \%, P=.013)$. HPV-positive primary tumors were more likely to be enhancing and exophytic with well-defined borders, whereas HPV-negative primary tumors were more likely to be isoattenuated and demonstrate ill-defined borders, though these results were not statistically significant. HPV-positive tumors were more likely to demonstrate cystic nodal metastases than HPV-negative tumors ( $36 \%$ versus $9 \%, P=.002)$.

CONCLUSIONS: In this matched and blinded analysis of the imaging differences between HPV-positive and HPV-negative oropharyngeal cancers, HPV-positive carcinomas often had primary lesions with well-defined borders and cystic nodal metastases, whereas HPV-negative primaries more often had poorly defined borders and invasion of adjacent muscle.

ABBREVIATIONS: HPV = human papillomavirus; SCCOP = squamous cell carcinomas of the oropharynx; EGFR = epidermal growth factor

W hereas age-adjusted incidence of oral cavity, laryngeal, and hypopharyngeal carcinomas has predictably declined with decreased smoking prevalence, there has been a paradoxical increase in the age-adjusted incidence of oropharyngeal carcinomas

Received November 9, 2012; accepted after revision January 9, 2013

From the Departments of Diagnostic Imaging (S.C.C., L.E.G.), Epidemiology (G.L., Q.W., E.M.S.), and Head and Neck Surgery (G.L., E.M.S.), University of Texas MD Anderson Cancer Center, Houston, Texas; Department of Radiology (S.C.C.), University of Texas Health Science Center, Houston, Texas; and Department of Otorhinolaryngology (B.W.P.), Mayo Clinic, Rochester, Minnesota.

This work was funded in part by start-up funds from University of Texas MD Anderson Cancer Center (E.M.S.); NIH Head and Neck SPORE grant P50CA097007 Career Development Award (E.M.S.); University of Texas MD Anderson Cancer Center Institutional Research Grant (E.M.S.); NIH grant K-12 88084 (E.M.S., faculty trainee; R.C. Bast, P.I.); NIH grant R03CA128110-01A1 (E.M.S.); NIH grant R01ES011740 (Q.W.); NIH grant R01CA131274 (Q.W.); and an NIH Cancer Center Support grant P30CA016672 (to John Mendelsohn)

Please address correspondence to Dr Lawrence E. Ginsberg, Department of Diagnostic Imaging, Unit 1482, University of Texas MD Anderson Cancer Center, 1400 Pressler St, Houston, TX 77030; e-mail: lginsberg@mdanderson.org

- Indicates open access to non-subscribers at www.ajnr.org

三 Indicates article with supplemental on-line tables.

http://dx.doi.org/10.3174/ajnr.A3524 secondary to the emergence of an epidemic of HPV-related squamous cell carcinomas of the oropharynx (SCCOP). ${ }^{1-5} \mathrm{HPV}$-positive SCCOP represents a unique demographic, molecular, and clinical entity with typically younger age of onset, limited tobacco exposure, and more favorable prognosis than HPV-negative SCCOP. ${ }^{6-10}$ The favorable prognosis among patients with HPVpositive SCCOP may be attributed in part to fewer somatic molecular alterations in HPV-positive cancers; HPV-positive smokers, however, appear to have a worse prognosis than HPV-positive nonsmokers, which may also be secondary to somatic molecular alterations in these cancers in smokers. ${ }^{6,11}$ It also appears that HPV-positive oropharyngeal carcinomas are a distinct histologic entity exhibiting basaloid, lymphoepithelial, and poorly differentiated histology as opposed to the keratinizing histologies seen in HPV-negative SCCOP. ${ }^{12}$

Although differences in the clinical characteristics, risk factors such as smoking and sexual behaviors, tumor pathways, and patient prognosis of HPV-positive versus HPV-negative SCCOP have been reported, distinctions between pretreatment imaging characteristics of HPV-positive and negative SCCOP have not 
been thoroughly investigated. ${ }^{7}$ The most comprehensive examination of the imaging characteristics of HPV SCCOP was performed by Goldenberg et al, ${ }^{13}$ whose retrospective review of pretreatment CT examinations of SCCOP demonstrated an association of cystic nodal metastases with base of tongue and tonsillar primary cancers and an association of cystic nodal metastases with HPV-positive cancers. Furthermore, Goldenberg et al made a distinction between cystic and necrotic nodal metastases, suggesting that they are distinct imaging and pathophysiologic entities. Cystic nodal metastases were defined as having homogeneous fluid content without internal complex, irregular, or solid areas and an enhancing capsule $<2 \mathrm{~mm}$ in thickness, whereas necrotic nodal metastases were defined as having thicker or more irregular walls with complex central low attenuation. ${ }^{13}$ Although the association of cystic nodal metastases with squamous cell carcinomas of the Waldeyer ring has been well established, ${ }^{14-17}$ our objective was to explore differences in the pretreatment imaging characteristics of HPV-positive and -negative oropharyngeal cancers.

\section{MATERIALS AND METHODS}

Patients with newly diagnosed oropharyngeal cancer who had been prospectively enrolled in a molecular epidemiology study conducted between May 1995 and June 2008 were matched on T-category, tumor subsite (base of tongue or tonsil), and smoking status (never, former, or current smoker). This research was approved by the institutional review board. Patients eligible for the current study met the following criteria: newly diagnosed, previously untreated SCCOP (base of tongue or tonsil), resident of the United States, and age 18 years or older. Exclusion criteria included treatment of head and neck malignancy (conventional surgical management, radiation therapy, or chemoradiation) before CT examination. Patients were also excluded if contrast-enhanced pretreatment CT imaging was not available for review in PACS. Each pair's HPV status was determined by HPV in situ hybridization or HPV16 polymerase chain reaction testing. "Never-smokers" were defined as having smoked fewer than 100 cigarettes in their lifetime, with "former smokers" having quit smoking at least 1 year before their cancer diagnosis. "Current drinkers" were defined as having at least 1 alcoholic drink per week for at least 1 year and who were still drinking in this manner at the time of their presentation, whereas "former drinkers" were defined as those who had drunk alcoholic beverages in this manner in the past but had begun drinking less or stopped drinking at least 1 year before presentation.

Retrospective review of pretreatment CT examinations of paired patients with HPV-positive or HPV-negative SCCOP matched for T-category, tumor subsite, and smoking status was performed by a single neuroradiologist blinded to HPV status and clinical stage. T-category was determined clinically with clinical examination, direct laryngoscopy, and pretreatment imaging. All CT examinations were performed on 16- and 64channel scanner systems (various software platforms; GE Healthcare, Milwaukee, Wisconsin). Images were obtained after administration of intravenous contrast, $(125 \mathrm{~mL}, 60-\mathrm{sec}-$ ond delay) from the aortic arch to the orbital roof with the following imaging parameters (tube current 100-600 mA, tube voltage $120 \mathrm{kV}$, gantry rotation time $0.5-1$ second, pitch
0.9, 1.25-mm image thickness). All patients with dental fillings had an additional stack of axial images obtained to angle away from the fillings. Although contrast bolus timing and volume of contrast may modify conspicuity of the interface between the primary lesion and surrounding soft tissues, in all cases contrast enhancement was sufficient to identify and adequately evaluate the primary tumor.

Demographic and clinical characteristics, imaging characteristics of primary lesion, and imaging characteristics of metastatic nodal disease were compared by means of Fisher exact testing. The McNemar $\chi^{2}$ test was used for the matched-pair analysis. Variables compared for the primary lesion included visibility, well-defined versus ill-defined border, submucosal spread, invasion of adjacent muscle, enhancement, necrosis, and exophytic margins. Submucosal spread was defined as extension of mucosal lesion $>1 \mathrm{~cm}$ in depth with infiltration of the submucosal fat plane and spread parallel to the mucosal surface. Variables compared for nodal metastases included radiologic $\mathrm{N}$ stage, presence of cystic nodes, necrotic nodes, metastatic spread to node of Rouvier (lateral retropharyngeal lymph node), nodal enhancement, and extracapsular spread. Extracapsular spread was recognized by indistinct nodal margins, irregular nodal capsular enhancement, and infiltration into the adjacent perinodal fat or muscle. Cystic lymph nodes were defined as having a thin $(<2 \mathrm{~mm})$ enhancing capsule and homogeneous fluid content $(>70 \%$ with $\mathrm{HU}<20$ ), with no internal complexity.

\section{RESULTS}

Of 102 matched pairs (204 patients) initially identified, 34 matched pairs were excluded because for one or both members of the matched pair no pretreatment CT was available for review, yielding 68 matched pairs. Each pair's HPV status had been determined by HPV in situ hybridization (38 pairs) or HPV16 polymerase chain reaction (30 pairs) testing.

The demographic and clinical characteristics of the patients with HPV-positive or HPV-negative SCCOP are presented in Online Table 1. Patients with HPV-positive and HPV-negative SCCOP were similar in demographics and clinical characteristics; however, there was a higher proportion of males in the group with HPV-positive SCCOP $(P=.02$, On-line Table 1$)$. Patients were excluded from analysis of the primary lesion (Table 1 ) if one or both members of the matched pair had a primary tonsillar tumor subsite with tonsillectomy before CT examination (7 matched pairs). Small primary tumors could not be visualized on CT imaging in 14 patients with HPV-positive SCCOP and in 10 patients with HPV-negative SCCOP (Table 1), and these patients were also excluded from further comparison of primary tumor imaging characteristics. HPV-negative tumors were more likely to demonstrate invasion of adjacent muscle (26\% versus $6 \%, P=.013$; Table 1) (Fig 1). HPV-positive tumors were more likely to have enhancing, exophytic primary lesions with well-defined borders by CT imaging (Fig 2), whereas HPV-negative tumors were more likely to have isoattenuated primary lesions that demonstrated ill-defined borders, though these results did not reach statistical significance (Fig 3).

Patients were excluded from analysis of the metastatic nodal imaging (Table 2) if one or both members of the matched pair had prior excisional lymph node biopsy (9 matched pairs). In 6 pa- 
Table 1: Imaging characteristics of patients with primary tumor among those with HPV+ oropharynx cancer and matched patients with HPV - oropharynx cancer

\begin{tabular}{|c|c|c|c|c|c|}
\hline \multirow[b]{2}{*}{ Imaging Characteristic } & \multicolumn{2}{|c|}{$\begin{array}{l}\mathrm{HPV}+ \\
(n=61)\end{array}$} & \multicolumn{2}{|c|}{$\begin{array}{l}\text { HPV- } \\
(n=61)\end{array}$} & \multirow[b]{2}{*}{$P$ Value } \\
\hline & $N$ & $\%$ & $N$ & $\%$ & \\
\hline Primary lesion is visible & & & & & .362 \\
\hline Yes & 47 & 77.0 & 51 & 83.6 & \\
\hline No & 14 & 23.0 & 10 & 16.4 & \\
\hline Primary lesion is enhancing & & & & & .182 \\
\hline Yes & 37 & 78.7 & 34 & 66.7 & \\
\hline No & 10 & 21.3 & 17 & 33.3 & \\
\hline $\begin{array}{l}\text { Primary lesion demonstrates } \\
\text { necrosis }\end{array}$ & 8 & 17.0 & 7 & 13.7 & .651 \\
\hline Yes & 39 & 83.0 & 44 & 86.3 & \\
\hline No & & & & & \\
\hline $\begin{array}{l}\text { Primary lesion demonstrates } \\
\text { well-defined border }\end{array}$ & & & & & .067 \\
\hline Yes & 35 & 74.5 & 29 & 56.9 & \\
\hline No & 12 & 25.5 & 22 & 43.1 & \\
\hline Primary lesion is exophytic & & & & & .095 \\
\hline Yes & 14 & 29.8 & 8 & 15.7 & \\
\hline No & 33 & 70.2 & 43 & 84.3 & \\
\hline $\begin{array}{c}\text { Primary lesion demonstrates } \\
\text { submucosal spread }\end{array}$ & & & & & .667 \\
\hline Yes & 26 & 55.3 & 26 & 51.0 & \\
\hline No & 21 & 44.7 & 25 & 49.0 & \\
\hline $\begin{array}{c}\text { Primary lesion invades } \\
\text { adjacent muscle }\end{array}$ & & & & & .013 \\
\hline Yes & 3 & 6.4 & 13 & 25.5 & \\
\hline No & 44 & 93.6 & 38 & 74.5 & \\
\hline
\end{tabular}

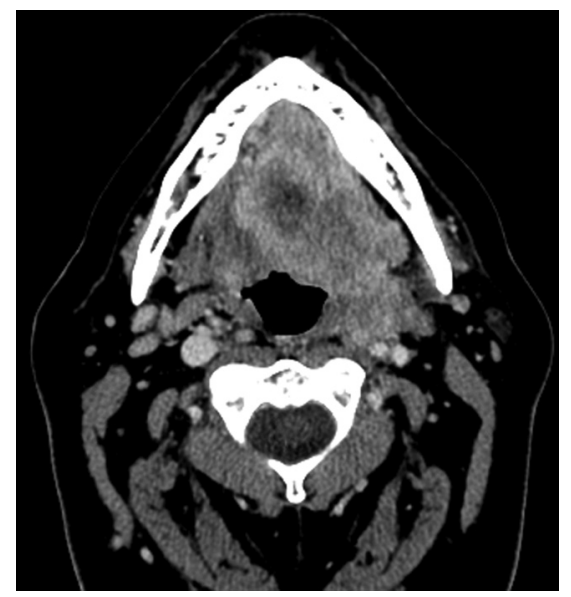

FIG 1. HPV-negative SCCOP demonstrating deep muscular invasion involving the extrinsic muscles of the tongue with submucosal spread. Axial contrast-enhanced CT image shows a large and deeply invasive T4 base of tongue lesion, extending anteriorly into the oral tongue.

tients with HPV-positive SCCOP and 9 patients with HPV-negative SCCOP, nodal metastases were not visible (Table 2). HPVpositive tumors were more likely to demonstrate cystic nodal metastases than HPV-negative tumors (36\% versus 9\%, $P=.002$; Table 2) (Fig 4).

In matched-pair analysis (On-line Table 2), 18 matched pairs were excluded from primary lesion subgroup analysis because one or both members of the matched pair had prior tonsillectomy or radiologic T0 category, and 14 matched pairs were excluded from nodal subgroup analysis secondary to one or both members having prior excisional lymph node biopsy or radiologic N0 category. In matched-

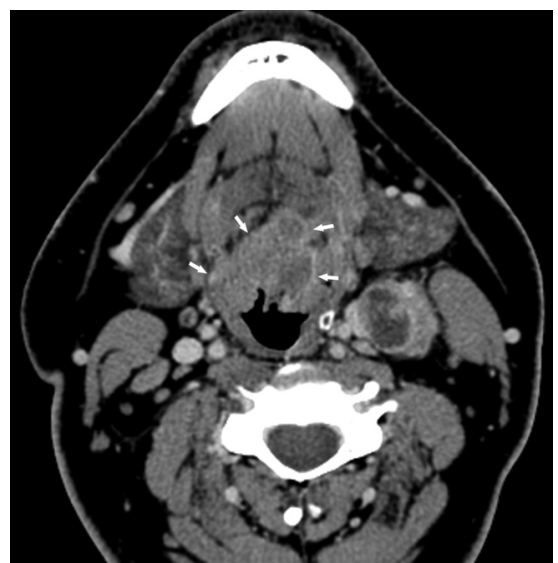

FIG 2. HPV-positive SCCOP with typically well-defined borders. Axial contrast-enhanced $\mathrm{CT}$ image shows a midline $\mathrm{T} 2$ base of tongue mass with well-defined margins (arrows). Level II left lymph node metastasis demonstrates extracapsular extension.

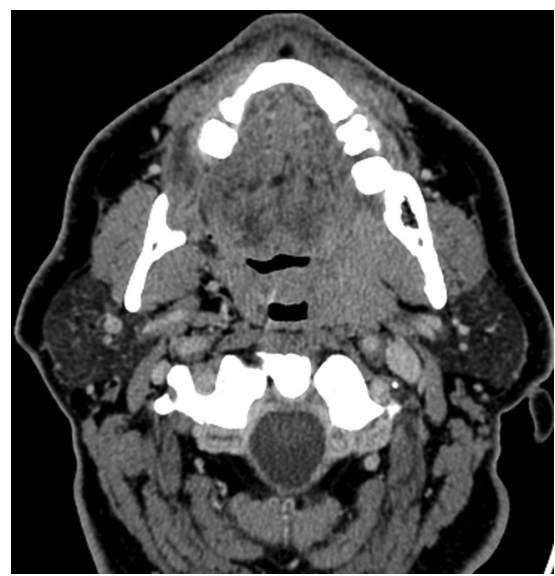

FIG 3. HPV-negative SCCOP demonstrating ill-defined borders. Axial contrast-enhanced CT image shows a mass in the left tonsil, with poorly defined borders, especially anteriorly.

pair analysis, cystic nodal metastases were associated with HPV-positive tumors ( $>5$-fold; $P=.013$; On-line Table 2$)$. Invasion of adjacent muscle was associated with HPV-negative tumors $(P=.002$; On-line Table 2).

\section{DISCUSSION}

In this blinded analysis of the CT imaging differences between HPV-positive and HPV-negative SCCOP, a greater percentage of HPV-positive SCCOP demonstrated primary tumors with welldefined borders whereas HPV-negative primary tumors demonstrated ill-defined borders and increased invasion of adjacent muscle. Despite the overall improved prognosis of HPV-positive SCCOP relative to HPV-negative SCCOP, we found no significant difference in incidence of extracapsular spread of nodal metastases, a well-established predictor of poor prognosis in squamous cell carcinoma of the head and neck. ${ }^{18-21}$ Our data are consistent with the findings of Goldenberg et $\mathrm{al},{ }^{13}$ which demonstrated increased incidence of cystic nodal metastases in HPVpositive SCCOP. These imaging differences between HPV-positive SCCOP compared with HPV-negative SCCOP are congruent with an understanding that HPV-positive SCCOP represent a 
Table 2: Imaging characteristics of nodal metastases among patients with HPV + oropharynx cancer and matched patients with HPV - oropharyngeal cancer

\begin{tabular}{|c|c|c|c|c|c|}
\hline \multirow[b]{2}{*}{ Imaging Characteristic } & \multicolumn{2}{|c|}{$\begin{array}{l}\mathrm{HPV}+ \\
(n=59)\end{array}$} & \multicolumn{2}{|c|}{$\begin{array}{l}\text { HPV- } \\
(n=59)\end{array}$} & \multirow[b]{2}{*}{$P$ Value } \\
\hline & $N$ & $\%$ & $N$ & $\%$ & \\
\hline Radiologic N stage & & & & & .754 \\
\hline No & 6 & 10.2 & 9 & 15.3 & \\
\hline N1 & 7 & 11.9 & 8 & 13.6 & \\
\hline N2 & 45 & 76.3 & 42 & 71.2 & \\
\hline N3 & 1 & 1.6 & 0 & 0 & \\
\hline $\begin{array}{l}\text { Nodal metastases demonstrate } \\
\text { extracapsular spread }\end{array}$ & & & & & .983 \\
\hline Yes & 16 & 30.2 & 15 & 30.0 & \\
\hline No & 37 & 69.8 & 35 & 70.0 & \\
\hline $\begin{array}{l}\text { Nodal metastases demonstrate } \\
\text { cystic morphology }\end{array}$ & & & & & .002 \\
\hline Yes & 19 & 35.8 & 5 & 10.0 & \\
\hline No & 34 & 64.2 & 45 & 90.0 & \\
\hline $\begin{array}{l}\text { Nodal metastases demonstrate } \\
\text { necrosis }\end{array}$ & & & & & 149 \\
\hline Yes & 37 & 69.8 & 41 & 82.0 & \\
\hline No & 16 & 30.2 & 9 & 18.0 & \\
\hline $\begin{array}{l}\text { Nodal metastases demonstrate } \\
\text { enhancement }\end{array}$ & & & & & 693 \\
\hline Yes & 33 & 62.2 & 33 & 66.0 & \\
\hline No & 20 & 37.8 & 17 & 34.0 & \\
\hline $\begin{array}{l}\text { Nodal metastases include node } \\
\text { of Rouvier }\end{array}$ & & & & & .474 \\
\hline Yes & 9 & 17.0 & 6 & 12.0 & \\
\hline No & 44 & 83.0 & 44 & 88.0 & \\
\hline
\end{tabular}

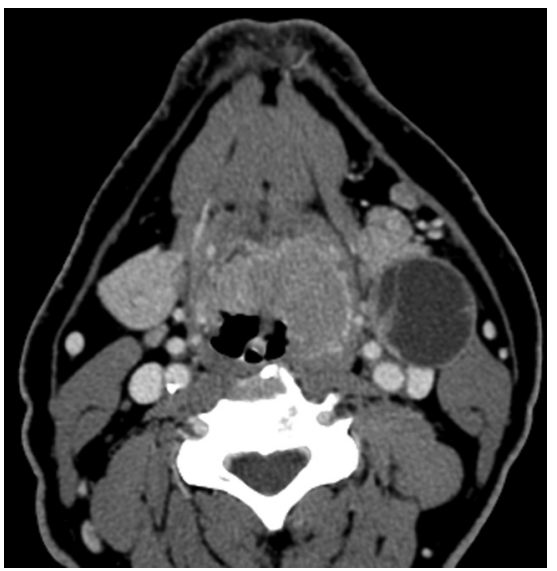

FIG 4. HPV-positive SCCOP demonstrating a cystic nodal metastasis. Axial contrast-enhanced CT image shows an obvious bulky midline left-sided base of tongue cancer (with characteristically well-defined borders) and a predominantly cystic mass in level Ila.

separate clinical entity, driven by different somatic molecular events and with a different clinical phenotype. ${ }^{11,22}$

Although HPV-positive SCCOP has a reliably better prognosis than HPV-negative SCCOP, deleterious somatic alterations resulting from tobacco exposure worsen the prognosis of HPV-positive smokers relative to HPV-positive nonsmokers. ${ }^{4} \mathrm{HPV}$ infection ultimately results $E 6$ and $E 7$ oncogene-mediated degradation of $\mathrm{p} 53$ and retinoblastoma ( $\mathrm{Rb})$ tumor suppressors. ${ }^{23}$ Overexpression of $\mathrm{p} 16$ protein, which functions as a tumor suppressor, is closely correlated with HPV positivity. ${ }^{4} \mathrm{HPV}$-positive nonsmokers are more likely to harbor an HPV-driven tumor phenotype characterized by high HPV titer, increased p16 expression, wild-type p53, and low epidermal growth factor (EGFR) expression. ${ }^{24}$ This molecular profile resulting from HPV positivity and limited or no smoking history results in favorable tumor phenotype and increased overall survival. ${ }^{4}$ Smokers are more likely to have tumors with adverse somatic alterations related to tobacco exposure, including mutated p53 and EGFR overexpression, concurrent with decreased HPV titer, and decreased p16 expression. $^{24}$

The most significant limitation of our study was our sample size. Several results lacking statistical significance in matched-pair analysis, including primary tumor borders and the presence of cystic lymph node metastases, are probably limited by a relatively small sample size. Despite limitations imposed by small sample size, matched-pair analysis provides a controlled and homogeneous setting and is a well-established means of controlling for confounding. Furthermore, all images were reviewed specifically for this study by a single radiologist without knowledge of the HPV status of the individual cases. Additional limitations of our study included retrospective review.

Finally, a common imaging finding in HPV-positive SCCOP, cystic metastases caused by small or poorly visualized primary, highlights a common clinical/radiologic pitfall that is of particular concern, given the rapidly rising incidence of SCCOP attributable to HPV. Because HPV-positive SCCOP is commonly clinically and radiologically occult at the primary site, radiologists must recognize that cystic neck masses in adult patients should be considered cystic metastases until proved otherwise; a brachial cleft cyst in an adult should be considered the exception rather than the rule for an adult with a cystic neck mass. Because HPV-positive SCCOP commonly arises in nonsmokers, a lack of significant tobacco or alcohol exposure does not provide evidence against malignancy. ${ }^{12,25}$ Such patients must be evaluated thoroughly by an otolaryngologist or head and neck surgeon, and the index of suspicion for carcinoma must be high.

\section{CONCLUSIONS}

HPV-negative primary tumors demonstrated ill-defined borders and increased invasion of adjacent muscle, whereas HPV-positive SCCOP more frequently demonstrated primary tumors with well-defined borders and cystic nodal metastases. Given the rising incidence of HPV-positive SCCOP, radiologists should maintain a high index of suspicion for underlying malignancy when evaluating cystic neck lesions in adult patients without significant tobacco or alcohol exposure.

\section{REFERENCES}

1. Chaturvedi AK, Engels EA, Anderson WF, et al. Incidence trends for human papillomavirus-related and -unrelated oral squamous cell carcinomas in the United States. J Clin Oncol 2008;26:612-19

2. Carvalho AL, Nishimoto IN, Califano JA, et al. Trends in incidence and prognosis for head and neck cancer in the United States: a sitespecific analysis of the SEER database. Int J Cancer 2005;114:806-16

3. Shiboski CH, Schmidt BL, Jordan RC. Tongue and tonsil carcinoma: increasing trends in the US population ages 20-44 years. Cancer 2005;103:1843-49

4. Sturgis EM, Ang KK. The epidemic of HPV-associated oropharyngeal cancer is here: is it time to change our treatment paradigms? J Natl Compr Canc Netw 2011;9:665-73

5. Sturgis EM, Cinciripini PM. Trends in head and neck cancer incidence in 
relation to smoking prevalence: an emerging epidemic of human papillomavirus-associated cancers? Cancer 2007;110:1429-35

6. Ang KK, Harris J, Wheeler R, et al. Human papillomavirus and survival of patients with oropharyngeal cancer. $N$ Engl $J$ Med 2010;363:24-35

7. Dahlstrom KR, Li G, Tortolero-Luna G, et al. Differences in history of sexual behavior between patients with oropharyngeal squamous cell carcinoma and patients with squamous cell carcinoma at other head and neck sites. Head Neck 2011;33:847-55

8. D'Souza G, Kreimer AR, Viscidi R, et al. Case-control study of human papillomavirus and oropharyngeal cancer. $N$ Engl $\mathrm{J}$ Med 2007;356:1944-56

9. Gillison ML, D'Souza G, Westra W, et al. Distinct risk factor profiles for human papillomavirus type 16-positive and human papillomavirus type 16-negative head and neck cancers. J Natl Cancer Inst 2008;100:407-20

10. Ji X, Neumann AS, Sturgis EM, et al. p53 codon 72 polymorphism associated with risk of human papillomavirus-associated squamous cell carcinoma of the oropharynx in never-smokers. Carcinogenesis 2008;29:875-79

11. Klussmann JP, Mooren JJ, Lehnen M, et al. Genetic signatures of HPV-related and unrelated oropharyngeal carcinoma and their prognostic implications. Clin Cancer Res 2009;15:1779-86

12. Marur S, D'Souza G, Westra WH, et al. HPV-associated head and neck cancer: a virus-related cancer epidemic. Lancet Oncol 2010;11:781-89

13. Goldenberg D, Begum S, Westra WH, et al. Cystic lymph node metastasis in patients with head and neck cancer: an HPV-associated phenomenon. Head Neck 2008;30:898-903

14. Flanagan PM, Roland NJ, Jones AS. Cervical node metastases presenting with features of branchial cysts. J Laryngol Otol 1994;108:1068-71

15. Gourin CG, Johnson JT. Incidence of unsuspected metastases in lateral cervical cysts. Laryngoscope 2000;110:1637-41
16. Regauer S, Mannweiler S, Anderhuber W, et al. Cystic lymph node metastases of squamous cell carinoma of Waldeyer's ring origin. Br J Cancer 1999;79:1437-42

17. Thompson LD, Heffner DK. The clinical importance of cystic squamous cell carcinomas in the neck: a study of 136 cases. Cancer 1998;82:944-56

18. Myers JN, Greenberg JS, Mo V, et al. Extracapsular spread: a significant predictor of treatment failure in patients with squamous cell carcinoma of the tongue. Cancer 2001;92:3030-36

19. Wenzel S, Sagowski C, Kehrl W, et al. The prognostic impact of metastatic pattern of lymph nodes in patients with oral and oropharyngeal squamous cell carcinomas. Eur Arch Otorhinolaryngol 2004;261:270-75

20. Iyer NG, Kim L, Nixon IJ, et al. Outcome of patients with early T1 and $\mathrm{T} 2$ squamous cell carcinoma of the base of tongue managed by conventional surgery with adjuvant postoperative radiation. Head Neck 2012 doi: 10.1002/hed.23071

21. Olsen KD, Caruso M, Foote RL, et al. Primary head and neck cancer: histopathologic predictors of recurrence after neck dissection in patients with lymph node involvement. Arch Otolaryngol Head Neck Surg 1994;120:1370-74

22. Martinez I, Wang J, Hobson KF, et al. Identification of differentially expressed genes in HPV-positive and HPV-negative oropharyngeal squamous cell carcinomas. Eur J Cancer 2007;43:415-32

23. Weinberger PM, Yu Z, Haffty BG, et al. Molecular classification identifies a subset of human papillomavirus-associated oropharyngeal cancers with favorable prognosis. J Clin Oncol 2006;24: $736-47$

24. Kumar B, Cordell KG, Lee JS, et al. EGFR, p16, HPV titer, Bcl-xL and p53, sex, and smoking as indicators of response to therapy and survival in oropharyngeal cancer. J Clin Oncol 2008;26:3128-37

25. Hudgins PA, Gillison M. Second branchial cleft cyst: not!! AJNR Am J Neuroradiol 2009;9:1628-29 